\title{
THE VISUALIZATION OF SHAFT VIBRATION USING THE ALGORITHM OF PHASE-AMPLITUDE DATA INTERPOLATION
}

\author{
Jacek Piątkowski \\ Institute of Computer and Information Sciences, Czestochowa University of Technology \\ Częstochowa, Poland \\ jacekp@icis.pcz.pl
}

\begin{abstract}
In many cases of observations of phenomena and physical processes we use data measured in multidimensional spaces. However, in practice, for the economic or technical reasons, we cannot obtain equally numerous data sets for all dimensions. In this case we can say that we are trying to assess or classify this process by using a few parts of its projection. For processing and computing of an input data a different solutions of interpolation and/or approximation are often used. But data, which have phase-amplitude nature, should be treated in a specific way. The lack of proper interpretation of phase shifts in many cases can result in obtaining the significant distortions of an analyzed process image. In this paper the algorithm of phase-amplitude interpolation is presented. This algorithm has been used for creating the contours charts, as projections of rotating shaft vibrations, in transient operating conditions of the machine.
\end{abstract}

Keywords: machine diagnostics, vibration condition monitoring, data interpolation, flexible rotor

\section{Introduction}

In a process of machine exploitation, especially critical machines, rightful identification and assessment of the current technical condition of monitored objects is significantly important $[1,2]$. The ability to track and classify current values of diagnostic features, which are the markers of a technical condition, lets us decide how the device should be operated. Effective and early identification of symptoms, characteristic for machine malfunctions, also allows for a significant safety improvement and reduces the operation cost of the monitored objects. It also helps to prevent breakdowns, which may cause serious consequences. For evaluation of the technical condition, a range of different measuring processes, such as thermal, electrical and tribological can be used. However, in most cases of constructions containing rotary elements, the best way to evaluate the technical condition is the usage of vibration parameters $[1,3,4]$. 
The application of absolute and relative vibrations measurements gives us the opportunity to diagnose the technical condition of monitored objects, as well as their individual components in a non-invasive way. Vibroacoustic signals are precisely related to characteristic, for these machines, parts or units operation frequencies such as shafts [5-7], gears [8], bearings [9-12] and so on. Thus, the measurement of for example absolute vibrations, apart from general evaluation of the technical state of examined machines, also allows us to identify irregularities in action of their individual components, caused by for instance unbalanced rotors, damaged bearings, gear tooth damage or misaligned couplings. The analysis of different properties of vibration signals - measured as a displacement of the shaft relative to the stationary bearing housing - allows us to identify journal bearings instabilities [13-16], shafts deformations, cracks or misalignment, unbalance, a rotor-to-stator rubbing on seals and bearings and other disturbances as well.

The vibration signals, as information carriers of both the work and technical condition of the machine, are processed to obtain specific features that characterise the monitored object. Generally these signals can be analyzed in the time domain, frequency domain, and time-frequency domain as well. The choice of number and type of symptoms used to identify the technical state of the machine usually depends on its construction and particular purpose, technical conditions and the mounting options of transducers and measurement systems. In the simplest case, one feature may be assessed - for example the RMS value of velocity, acceleration or displacement of the vibrations, the level of acoustic emission or the amount of shock pulses [9]. In the majority of advanced diagnostic systems, recorded vibration signals are processed in order to determine a number of measures, which represent the monitored objects in a multidimensional-non-orthogonal space called symptom space [1]. Used symptoms must be chosen on the basis of their current values, the classification of current technical state of the machine could be unequivocal. The set of possible technical states of the machine is infinite and uncountable. However, in practice, all the possible technical states of the machine are typically divided into a few classes. In the simplest case, they may be two classes - usable or unusable.

Very often, however, for economic as well as technical reasons, a corresponding set of features cannot be acquired. For instance, in the time and space dimensions, because practically always there are restrictions for the places where transducers can be mounted [5]. So, we cannot obtain equally numerous data sets for all dimensions. Thus we are trying to create an image which represents the observed process by using a few or over a dozen time series which contain a few hundred or a few thousand samples. In this case we can say that we are trying to assess or classify this process by using a few parts of its projection. Sometimes however, such conduct may result in obtaining false results.

In the processing of measuring signals we often use various kinds of interpolation and/or approximation methods to create an image of examined process or to estimate missing values in order to obtain results that cannot be determined directly. For example George, Petros et al. in [12] presented a method for the extraction of diagnostic representations of vibration recordings using the Symbolic Aggregate 
approXimation (SAX) framework and related intelligent icons representation. However, sets of symptoms, the values of which change in a phase-amplitude way, must be treated in a specific way. The lack of proper interpretation of phase shifts in many cases can result in obtaining the significant distortions of an analyzed process image. An example of the phenomena, where amplitude and phase information must be fully and correctly utilised, is vibrations of a flexible rotor shaft [5-7].

Simultaneous assessment of the value of relative amplitude and the relative phase of these vibrations allows for the identification of the shape shaft vibration form, so-called the vibration modes. The example of time waveforms of signals recorded (using four non-contact proximity transducers) for the first most common two shaft vibration modes is shown in Figure 1.
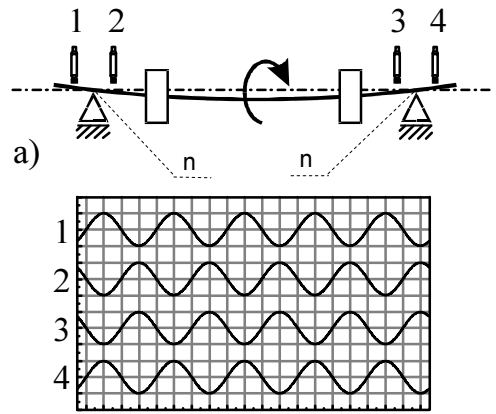
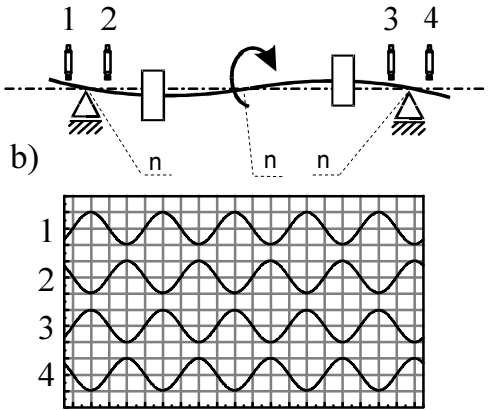

Fig. 1. The theoretical waveforms of the shaft relative vibration: a) first mode vibration, b) second mode vibration

Assuming a perfectly rigid bearing support, nodes (n) of flexural vibration exist in shaft support points. As we can see, transducers mounted on opposite sides of the bearing support record signals shifted in phase by $180^{\circ}$. For the first mode of vibration - Figure 1a we can observe the phase compliance between the signals recorded in channels 1,4 and 2, 3. A different situation exists for the second vibration mode - Figure $1 \mathrm{~b}$ where the signals 1, 4 and 2, 3 are in athi-phase.

In the case of the model example presented in Figure 1 occurs no ambiguity or difficulty in interpreting features representing examined process. However in the case of real technical objects we cannot always acquire such clear measurement results. Often, when a machine is operating under steady state conditions, the identification of only a form or level of vibrations is not enough for correct classification of its technical state. For most complex technical objects, such as turbogenerators, the identification of changes in their technical condition is possible as a result of the assessment of startup and shutdown characteristics (images), that is created in transient operating conditions [5, 7, 13-16]. Then the comparison is made on the current image (created by the defined space of selected features) with the same image made earlier, when the state machine was recognized as correct. 
Identification of deviation of the analyzed image from its pattern provides us the information about the change of the technical condition.

This study presents results of the application of the method (developed by the author) of phase-amplitude interpolation of measuring signals for creating three-dimensional images of relative shaft vibrations of rotating machinery.

\section{Measurement setup and data acquisition}

For testing the phase-amplitude interpolation algorithm discussed in the study, experimental data delivered from the model of the rotating machine were used. During the experiment, by using proximity transducers, four relative shaft vibration and keyphasor signals were recorded. Data acquisition was carried out with successively changed rotor speeds - from 500 to $8000 \mathrm{rpm}$, with an increment of $100 \mathrm{rpm}$. In Figure 2 the scheme of the used measuring system is displayed.

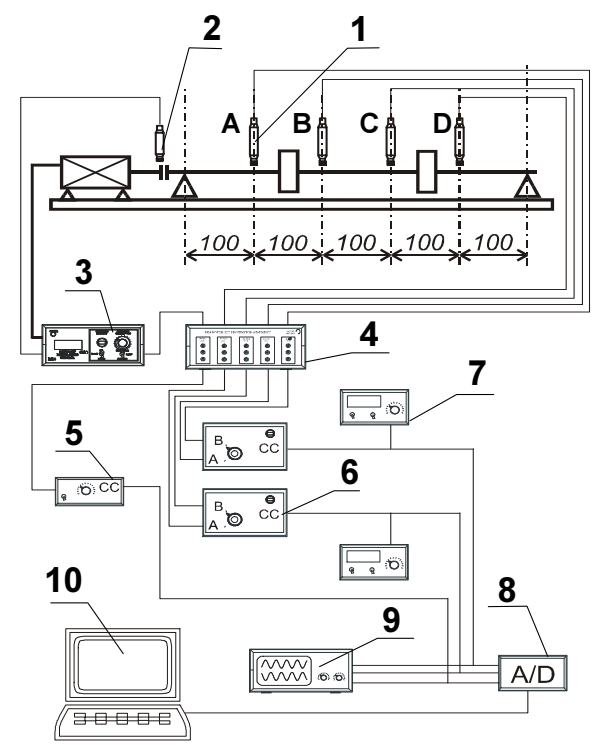

Fig. 2. The experimental setup: 1 - proximitors, 2 - keyphasor, 3 - engine rotation speed controller, 4 - Bently Nevada RK4 Rotor Kit Proximitor Assembly, 5 - signal conditioner, 6 - signal conditioner and analog low-pass filter,

7 - voltmeter, 8 - A/D converter, 9 - oscilloscope, 10 - computer

Four proximitors - Figure 2 (1) - to measure shaft relative vibration were placed vertically in four equidistant measuring planes $(\mathrm{A}, \mathrm{B}, \mathrm{C}, \mathrm{D})$ located between two bearing supports (apart by $500 \mathrm{~mm}$ ). The recorded voltage signals (proportional to the value of vibration displacement) were analog processed by the signal conditioner. Low-pass filtration was performed $\left(\mathrm{f}_{\mathrm{N}}=800 \mathrm{~Hz}\right)$ as well the change of the value of the DC component and adequate amplification of the signal - in order to 
adjust voltage to the range of the input voltage of 12-bit analog-to-digital converter. Sampled vibration signals were recorded in computer memory.

\section{The assessment of possibilities of visualization and measurement data analysis}

As a result of executed experiment, a database of 152 files containing time series of sampled measurement signals was obtained. Each of those files contain two vibration signal waveforms (from two neighbouring transmitters) and phase marker signal waveform, which makes it possible to recreate later both relative and absolute phase registered vibrations.

The examples of signal time series are presented in Figure 3, which depicts changes in the value of the amplitude of the vibration displacement (d) as a function of time (t) per one rotation of the shaft with period $(\mathrm{T})$.

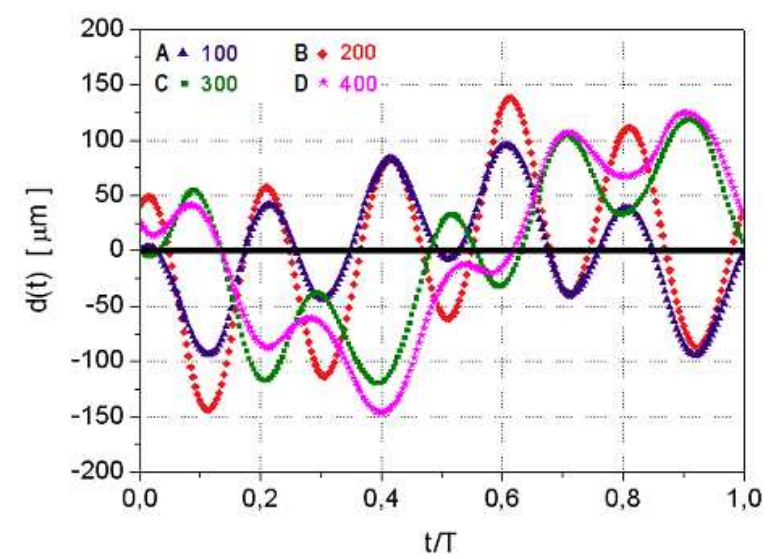

Fig. 3. The waveforms of relative shaft vibration (at $500 \mathrm{rpm}$ )

As we can see, the dominant components of analyzed vibrations are the first two harmonics - (1X and 2X). Signals from the transducers A and B are in anti-phase to the signals from the transducers $\mathrm{C}$ and $\mathrm{D}$. Given the construction of the machine from which the measurement data came, we could (wrongly) suppose that it is the second mode of shaft vibration, similar to that shown in Figure $1 \mathrm{~b}$. Wrongly, because later the first and second critical speeds were identified - near $2400 \mathrm{rpm}$ and near $5700 \mathrm{rpm}$.

The presentation of measurement data in the form of charts presented in Figure 3 does not allow for the comprehensive assessment of the spatial shape of the shaft vibration. Even in the case of an uncomplicated model of the machine, from the analyzed charts, it is difficult for example to assess an asymmetry of the shaft deflection. The real industrial objects, such as a turbogenerators, usually have shafts of different diameters. The measurement points are localized mostly in the bearing housings, which can be placed differently along the axis of the shaft. Because 
of that, the correct observation of the waveforms in such form as presented in Figure 3 is not possible.

The application of direct presentation of vibration waveforms in a three-dimensional coordinate system (as presented in Fig. 4) does not produce better results. The image of the studied vibration process created in this way is difficult to analyze, and even impossible to interpret correctly. The number of vibration waveforms limited by the number of measuring points prevents one from the direct execution of the contours chart. In fact, we have the data delivered from far different resolutions in time and space. In this case we are talking about 6 values of the space vector, and 512 values of the vector of time. That is because we have four time series of vibration displacement, measured in places A, B, C and D (Fig. 2), and two time series of zero values on bearing supports. Each of the time series contains 512 samples.

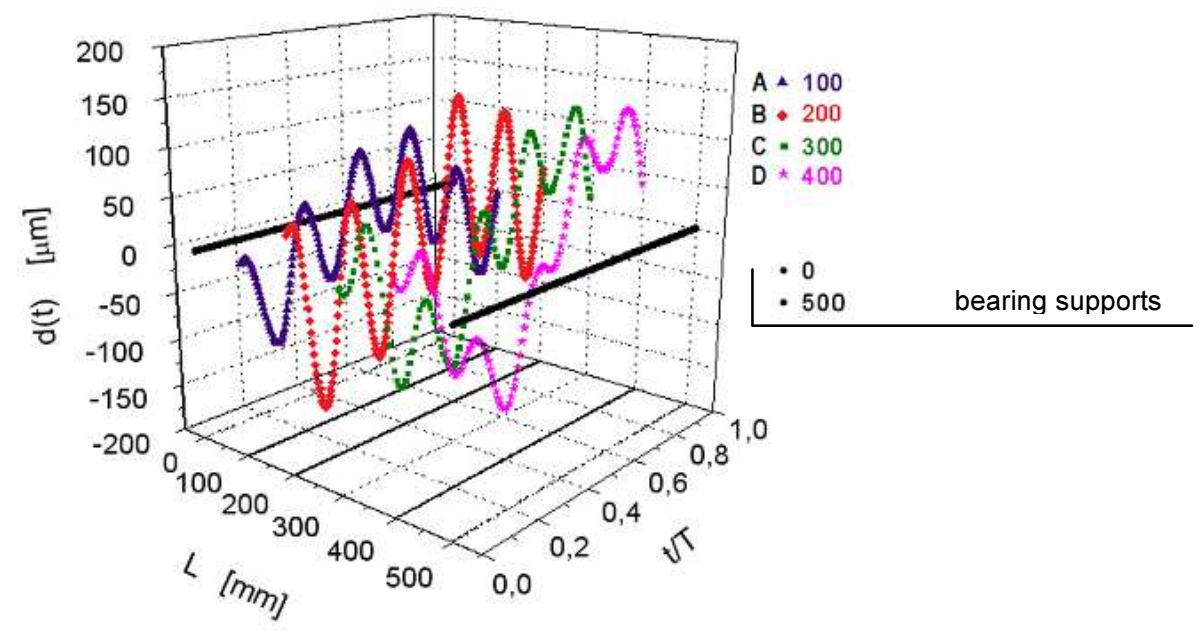

Fig. 4. The waveforms of relative shaft vibration (at $500 \mathrm{rpm}$ )

The application of data interpolation methods, which does not include the presence of phase shifts between processed signals, deforms the image of the analyzed process. The example of image distortion can be seen on the contours charts, which are presented in Figures 5 and 6 . These charts were prepared on the basis of the same data sets as in the case of Figures 3 and 4, using interpolation methods of the Origin Pro 7 program.

In the first case (Fig. 5a) a correlation method was used. As we can see, this chart gives only a very general projection of the vibration state of the examined object. This is because we can roughly determine the direction of shaft deflection and perceive asymmetry of these deflections. But this image is practically not suitable for identifying changes in the technical condition of the machine. As a result of processing of the input data, the signals components with frequencies higher than the first harmonic (1X) has been virtually eliminated. 
a) $d(t)$

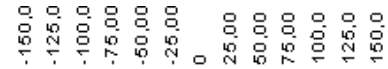
[ $\mu \mathrm{m}$ ]

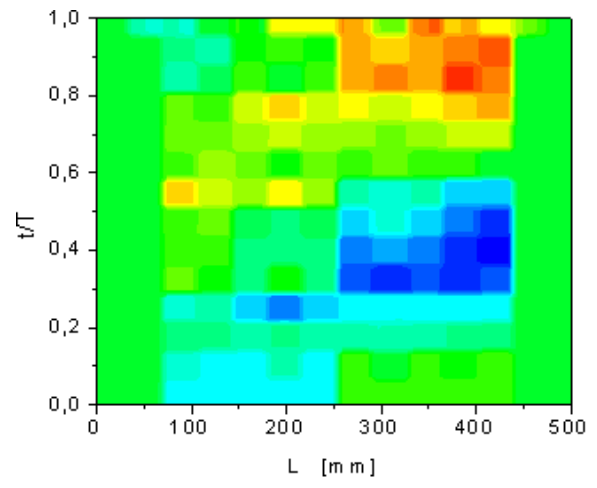

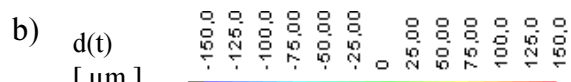

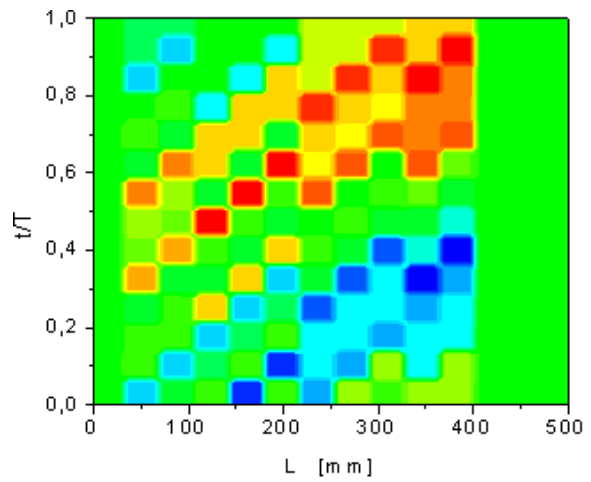

Fig. 5. The contours charts of relative shaft vibration (at $500 \mathrm{rpm}$ ): a) the correlation method of data interpolation, b) the weighted arithmetic means of data interpolation

A similarly bad effect was obtained using a weighted arithmetic means interpolation method (Fig. 5b). Also, in this case, the image of the examined process is very imprecise and practically devoid of information carried by signal components higher than $1 \mathrm{X}$.

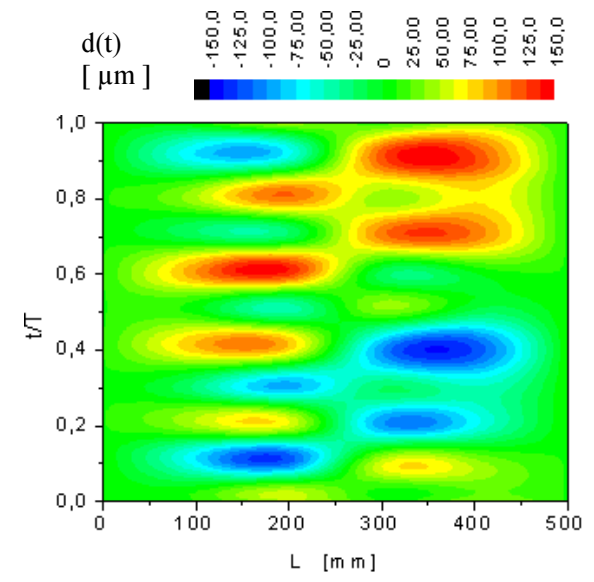

Fig. 6. The contours chart of relative shaft vibration (at $500 \mathrm{rpm}$ ) the Renka Cline method of data interpolation

The relatively best result was obtained using Renka Cline method (Fig. 6) because the two first harmonics are now visible. But also in this case, the created image can be rather treated as a demonstration. 


\section{The phase-amplitude interpolation}

As previously mentioned, too large a disparity between the number of elements acquired in the space domain and elements from the time domain does not allow for the direct creation of contours charts, which correctly shows the image of the vibration condition of the shaft of rotating machinery. We also saw that a lack of proper interpretation of phase shifts in the processing of the measurement signals led to a significant distortion of the projection of the analyzed process. However, if the signals from neighbouring measuring planes are correlated, we can make an assumption of possible use of the phase-amplitude data interpolation for elimination of previously mentioned restrictions.

The algorithm described below further assumes that both the amplitude and the phase between the neighboring time series of the data changes in a linear way. In general, however, the algorithm could be adapted to use the other functions, interpolating the changes of amplitude and phase.

\subsection{The algorithm of phase-amplitude interpolation}

The algorithm of phase-amplitude interpolation is based on the assumptions that:

- input data series are the representation of the process, which have a periodical nature and are synchronously sampled;

- there is a possibility to determine the function of correlation between neighboring time series of the data.

So, if we have $i=1,2, \ldots, I(I \geq 2)$ time series recorded in $i$ measurement places, the one step of data interpolation (using real data from places $i$ and $i+1$ ) can be described as below:

1. The covariance function is being calculated on the basis of waveforms $x_{l i}(t)$ and $x_{l i+1}(t)$ coming from the neighboring measurement points

$$
\hat{c}_{k}=\frac{1}{N} \sum_{t=1}^{N-k}\left(x_{l_{i}, t}-\bar{x}_{l}\right)\left(x_{l_{i+1}, t+k}-\bar{x}_{l_{i+1}}\right)
$$

where:

$N$ - number of samples of processed time series,

$k$ - correlation distance, $(k=0,1,2, \ldots, K)$ and $K \leq N / 4$,

$l_{i}$ - the $i$-th spatial coordinate.

2. The maximum value of the covariance function $\hat{c}_{k}$ is being found. The correlation distance $k$, when the covariance function $\hat{c}_{k}$ reaches its maximum value is interpreted as the phase shift angle between the used measurement signals (as a relative phase, which assumes values between $0 \pm \pi$ ).

3. A shift of $x_{l_{i+1}, t}$ signal is being made by the value of the phase angle $k$ according to the relation: 


$$
x_{l_{i+1}, t}^{\prime}=x_{l_{i+1}, t-k}
$$

where: $k$ assumes values $0 \pm n s / 2$, where $n s$ is the number of samples per one period of the fundamental signal component (1X).

4. On the basis of corresponding values $x_{l_{i}, t}$ and $x_{i_{i+1}, t}^{\prime}$ the values $x_{\lambda_{j, t}}^{j}$ are calculated:

$$
x_{\lambda_{j}, t}^{j}=g\left(\lambda_{j}\right)
$$

where:

$\lambda_{j} \quad$ - the $j$-th spatial coordinate from the range $l_{i} \div l_{i+1}$,

$g\left(\lambda_{j}\right)$ - the interpolation function which coefficients basing on $x_{l_{i}, t}$ and $x_{l_{i+1}, t}^{\prime}$ values was calculated.

5. For the next $(j)$ time series, which values were calculated in step 4 the phase shift is made:

$$
x_{\lambda_{j, t}}^{j}=x_{\lambda_{j}, t+\xi}^{j},
$$

where:

$$
\xi=\frac{k\left(l_{i+1}-l_{i}\right)}{\lambda_{j}-l_{i}} .
$$

The illustration of operation of the discussed algorithm is shown in Figure 7. Time series marked with symbols $x_{l_{i}, t}$ and $x_{l_{i+1}, t}$ are the result of sampling measurement data. We can see that relative phase between signals has a value of about $3 / 16 \pi$. Therefore, based on the assumption of linear changes of the amplitude and phase between waveforms coming from neighboring measuring points, it is possible to generate virtually any number of waveforms for mediate coordinates. In Figure 7 waveforms generated by the help of the described algorithm are marked with thinner lines.

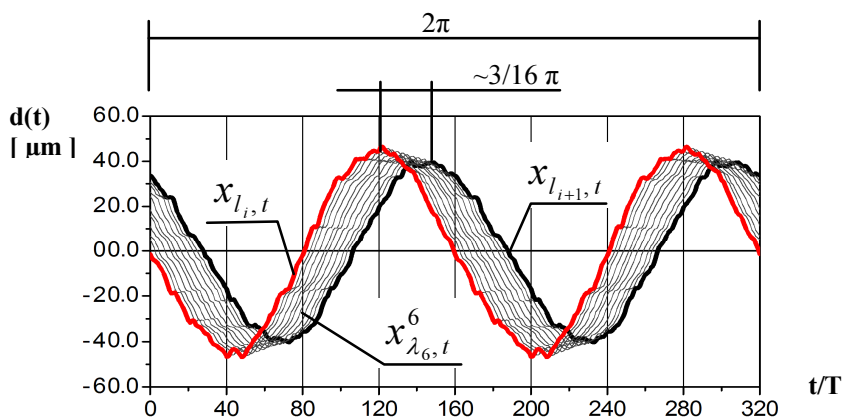

Fig. 7. Illustration of the operation of the phase-amplitude interpolation algorithm 


\subsection{The results of the algorithm application}

The application of the algorithm for measurement data interpolation discussed above has helped to achieve significant improvement of contours charts quality, which reflect the condition of the analyzed vibration process. Examples of images created in this way are shown in Figures 8 and 9.

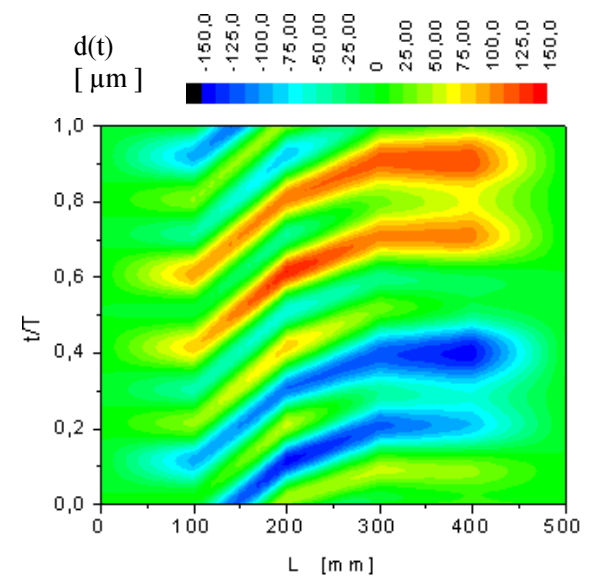

Fig. 8. The contours chart of relative shaft vibration (at $500 \mathrm{rpm}$ ).

The algorithm of phase-amplitude data interpolation

Figure 8 shows the image of the shaft vibration state at speed $500 \mathrm{rpm}$. It was, therefore, created based on the same input data sets like in the case of charts presented in Figures 3 to 6 . It shows the clear dominance of the first two vibration harmonics and asymmetry in shaft deflections. We can see clearly how the phase (phase lag) of the shaft vibration is changing with increasing distance from the coupling.

Figure 9 illustrates the vibration state of the shaft at the speed $2400 \mathrm{rpm}$ and $5700 \mathrm{rpm}$, which, as we can see, is quite different than shown in Figure 8. In the first case (Fig. 9a) the amplitude of shaft vibration, in which only the basic component is present, is almost six times greater than the previous one. Signals from all measuring points practically have phase compliance. It is seen that signals from $\mathrm{C}$ and $\mathrm{D}$ localization are slightly ahead of the signals from proximitors mounted at points B and A. Without any problem we can identify the resonance nature of the current vibrations, with the presence of the first vibration mode (like in Fig. 1a). Figure 9b illustrates the image of shaft vibration at the speed $5700 \mathrm{rpm}$. As at the speed of $2400 \mathrm{rpm}$ also in this case, the dominant is the first harmonic vibration, but the amplitudes are half the size. There is no problem in identifying the second modal shape of shaft vibration, with the characteristic phase shift of $180^{\circ}$. 
a)
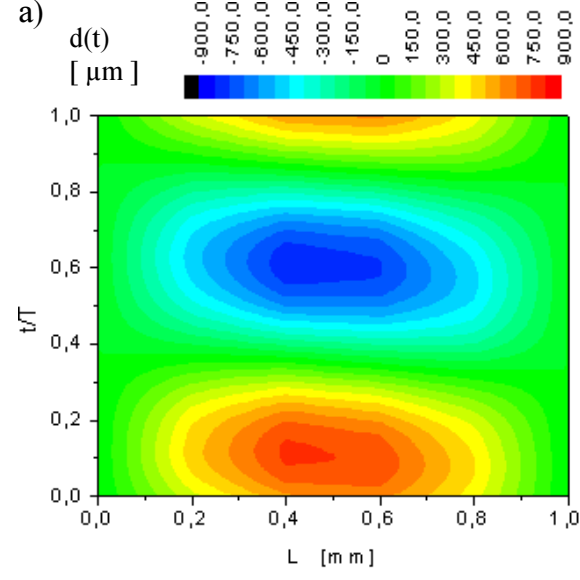

b)
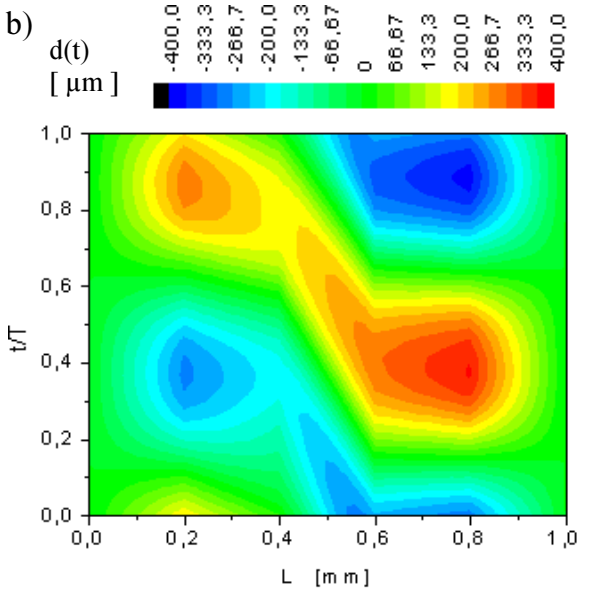

Fig. 9. The contours charts of relative shaft vibration created using the algorithm of phase-amplitude data interpolation: a) $2400 \mathrm{rpm}$, b) $5700 \mathrm{rpm}$

\section{Conclusions}

The results obtained during the research allow us to conclude that the described method turns out to be an effective tool which allows us to interpolate measurement sets representing phenomena which have a phase-amplitude nature. Despite the two orders of magnitude of difference between the number of data obtained in the domain of time and space, the use of phase-amplitude interpolation allowed us to level those disproportions by making it possible to create the contours charts of the analyzed vibration process.

The application of the contours charts to assess the vibration state of the shaft allowed us to increase the possibility of identifying changes in the technical state of the monitored object. The application of the contours charts to assess the vibration state of the shaft allowed us to increase the possibility of identifying changes in the technical state of the monitored object. It can be done by marking the acceptability areas (typical for monitored machine) for both the amplitude and phase of shaft vibration. The same technique can be implemented to analyse other processes which have phase-amplitude nature.

\section{References}

[1] Cempel C., Multidimensional condition monitoring of mechanical systems in operation, Mechanical Systems and Signal Processing 2003, 17(6), 1291-1303.

[2] Ziopaja K., Pozorski Z., Garstecki A., Damage detection using thermal experiments and wavelet transformation, Inverse Problems in Science and Engineering 2011, 19(1), 127-153.

[3] Karabay S., Uzman I., Importance of early detection of maintenance problems in rotating machines in management of plants: Case studies from wire and tyre plants, Engineering Failure Analysis 2009, 16, 212-224. 
[4] Cempel C., Application of TRIZ approach to machine vibration condition monitoring problems, Mechanical Systems and Signal Processing 2013, 41, 328-334.

[5] Vania A., Pennacchi P., Experimental and theoretical application of fault identification measures of accuracy in rotating machine diagnostics, Mechanical Systems and Signal Processing 2004, $18,329-352$.

[6] Liu S., A modified low-speed balancing method for flexible rotors based on holospectrum, Mechanical Systems and Signal Processing 2007, 21, 348-364.

[7] Pennacchi P., Vania A., Accuracy in the identification of a generator thermal bow, Journal of Sound and Vibration 2004, 274, 273-295.

[8] Qingbo He, Zhihua Feng, Fanrang Kong, Detection of signal transients using independent component analysis and its application in gearbox condition monitoring, Mechanical Systems and Signal Processing 2007, 21, 2056-2071.

[9] Idriss El-Thalji, Erkki J., A summary of fault modelling and predictive health monitoring of rolling element bearings, Mechanical Systems and Signal Processing 2015, 60-61, 252-272.

[10] Yi W., Guanghua X., Qing Z., Dan L., Kuosheng J., Rotating speed isolation and its application to rolling element bearing fault diagnosis under large speed variation conditions, Journal of Sound and Vibration 2015, 348, 381-396.

[11] Tuncay K., Nizami A., Experimental diagnostics of ball bearings using statistical and spectral methods, Tribology International 2009, 42, 836-843.

[12] George G., Petros K., Theodoros L., Chrysostomos D.S., Rolling element bearings diagnostics using the Symbolic Aggregate approXimation, Mechanical Systems and Signal Processing 2015, 60-61, 229-242.

[13] Helio Fiori de Castro, Katia Lucchesi C., Rainer N., Whirl and whip instabilities in rotor-bearing system considering a nonlinear force model, Journal of Sound and Vibration 2008, 317, 273-293.

[14] Robbersmyr K.G., Olsen H., Reza Karimi H., Tonder K., Oil whip-induced wear in journal bearings, Int. J. Adv. Manuf. Technol. 2014, 73, 973-980.

[15] Chen-Chao F., Min-Chun P., Experimental study on the whip elimination of rotor-bearing systems with electromagnetic exciters, Mechanism and Machine Theory 2011, 46, 290-304.

[16] Chen-Chao F., Jhe-Wei S., Min-Chun P., Wen-Chang T., Study of start-up vibration response for oil whirl, oil whip and dry whip, Mechanical Systems and Signal Processing 2011, 25, 3102-3115. 